\title{
Application of laser speckle flow graph for compensatory sweating
}

Hidehiro Yamamoto, MD, and Masayoshi Okada, MD, Tokyo, Japan

From the Department of Surgery, Yamamoto-Hidehiro Clinic, Tokyo, Japan.

Disclosures: Authors have nothing to disclose with regard to commercial support.

Received for publication April 14, 2017; revisions received May 25, 2017; accepted for publication May 31, 2017; available ahead of print June 27, 2017.

Address for reprints: Hidehiro Yamamoto, MD, Department of Surgery, Yamamoto-Hidehiro Clinic, 2-28-4 Dogenzaka, Shibuya-ku, Tokyo 150-0043, Japan (E-mail: bestets@yahoo.co.jp).

J Thorac Cardiovasc Surg 2017;154:e113-5

$0022-5223 / \$ 36.00$

Copyright (C) 2017 by The American Association for Thoracic Surgery

http://dx.doi.org/10.1016/j.jtcvs.2017.05.091

$\square$ Video clip is available online.

Laser speckle flow graph (LSFG) devices enables the noninvasive evaluation of skin blood perfusion. The laser speckle phenomenon is an interference event observed when coherent light sources, such as lasers, are scattered by a diffusing surface. The speckle pattern appears in accordance with blood flow velocity. ${ }^{1,2}$ Herein, we applied LSFG techniques to treat compensatory sweating (CS) (Video 1).

\section{CASE REPORT}

A 34-year-old man with facial and axillar hyperhidrosis underwent bilateral endoscopic thoracic sympathectomy (ETS) of the both sides 7 years previously. Transection of the sympathetic chain was then performed on the second rib. After 1 month, he complained of CS on the chest, back, and abdomen. Furthermore, recurrence of facial sweating was recognized on the left side 6 years after the ETS (Figure 1, $A-C$ ). To treat the recurrence and CS, we used an LSFG device (LSFG-ANW; Soft Care Co Ltd, Fukuoka, Japan) to detect the sympathetic nerve causing the CS. To determine which ganglion to remove, transient changes in the LSFG were monitored while each sympathetic nerve was stimulated with an electrosurgical unit (50-400 V, 3-30 kHz, MS7000AS; Senko Medical Manufacturing Co. Ltd, Tokyo, Japan).

After we identified the sympathetic nerves causing a change in the LSFG (Figure 2, B, Video 2) corresponding to the same area of the skin where the left-sided CS appeared (the third and sixth ganglions), resections of the left second, third, and sixth ganglions were performed. Sweating of the left face decreased, and CS of the left side improved (Figure 1, E-G). After 1 year, resections of the right third, fourth, sixth, and seventh ganglion were performed in the a similar manner with LSFG. CS on the right side improved (Figure 1,D and $H$ ). During 4 years of

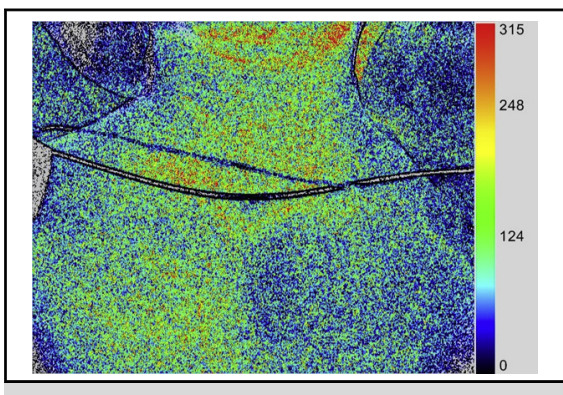

Compensatory sweating after thoracic sympathectomy was decreased by resympathectomy.

\section{Central Message}

The sympathetic nerve causing compensatory sweating can be identified by the use of laser speckle flow graph techniques. Removing the relevant nerve ameliorates compensatory sweating.

See Editorial Commentary page e117.

follow-up, there has been no re-recurrence and no new CS has appeared.

\section{COMMENT}

Local hyperhidrosis is thought to be caused by increased sympathetic signaling to the sweat glands. ETS is thought to divide the signaling. Although CS after ETS has been considered to be a thermoregulatory response to the anhidrosis caused by the ETS, ${ }^{3-5}$ it is quite doubtful that the large

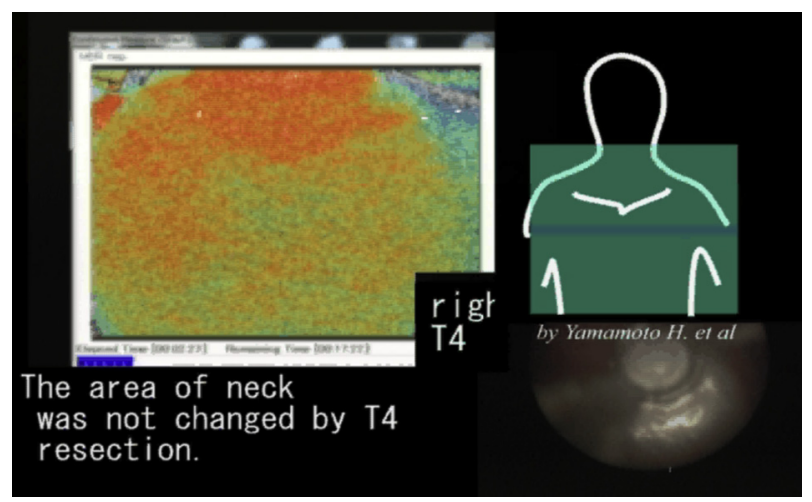

VIDEO 1. A transient decrease in the skin blood perfusion was seen repeatedly even after resecting the sympathetic trunk (another patients). Video available at: http://www.jtcvsonline.org/article/S0022-5223(17) 31157-1/fulltext. 


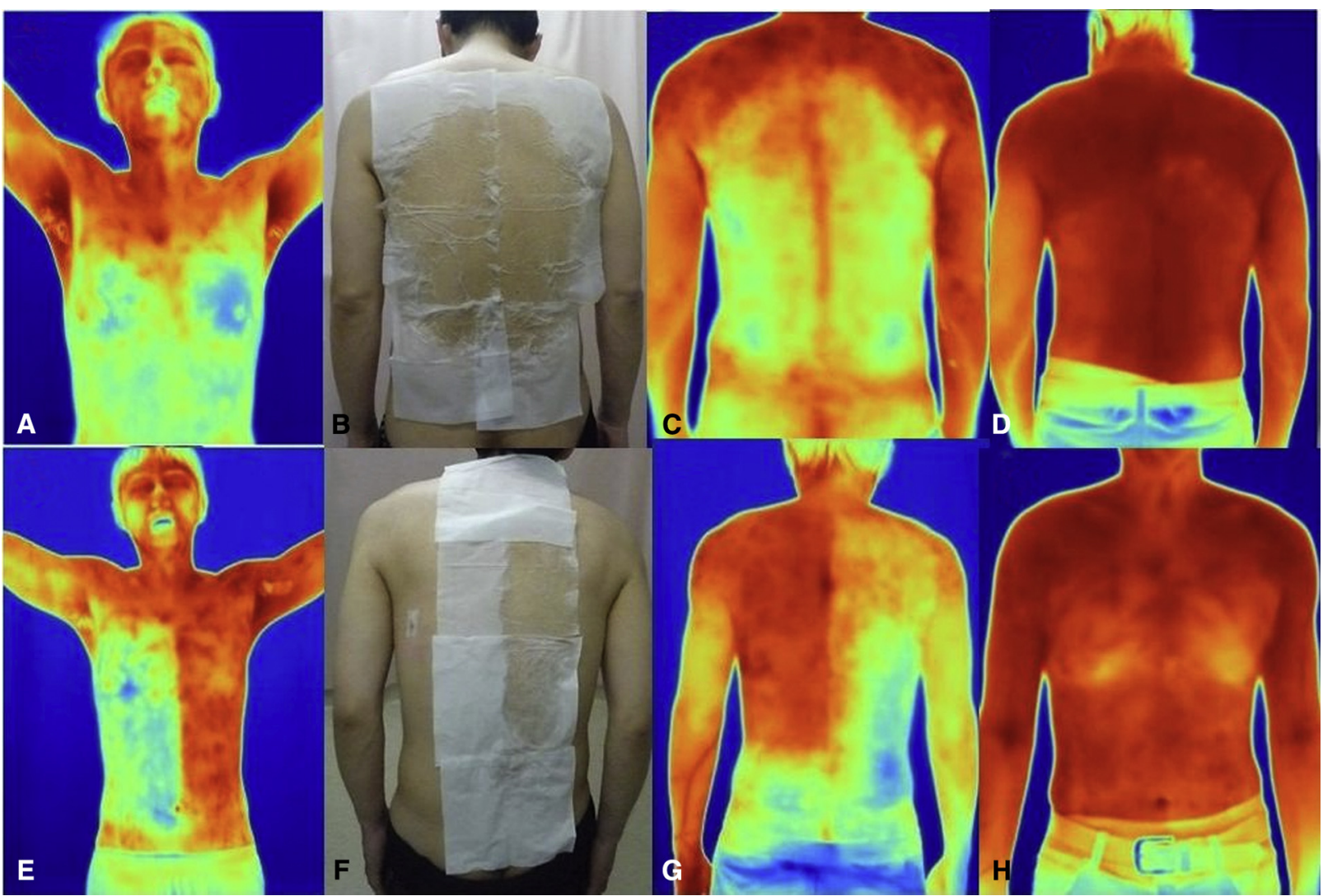

FIGURE 1. Sweating and skin temperature after jogging for 20 minutes. A, Thermography of the front of the body. A low skin temperature was recorded on part of the precordial region and the left face. B, Sweat state on the back; clear boundary line can be seen. C, Thermography of the back. A low skin temperature was recorded on the back. D, Thermography of the back after bilateral treatment of CS. A low skin temperature was not recorded on the back. E, Thermography of the front of the body. The low skin temperature on the part of the left precordial region and sweating of the left face is no longer observed. F, Sweat state on the back after treatment of the left side for CS. The decrease in sweating of the left side is substantial. G, Thermography of the back. H, Thermography of the back after bilateral treatment for CS. The skin temperature has returned to normal.

quantity of sweat seen in CS helps to control temperature.

Nervous system changes may lead to a large quantity of signals to the skin where CS appears. By dividing one of the nerve circuits, $\mathrm{CS}$ can be ameliorated. The 2 functions of the sympathetic nerves (vasomotor action and sudomotor actions) work in the same skin domain. The vasomotor response of the arteriole in the skin can be observed in the LSFG. When the sympathetic nerve is stimulated, a change

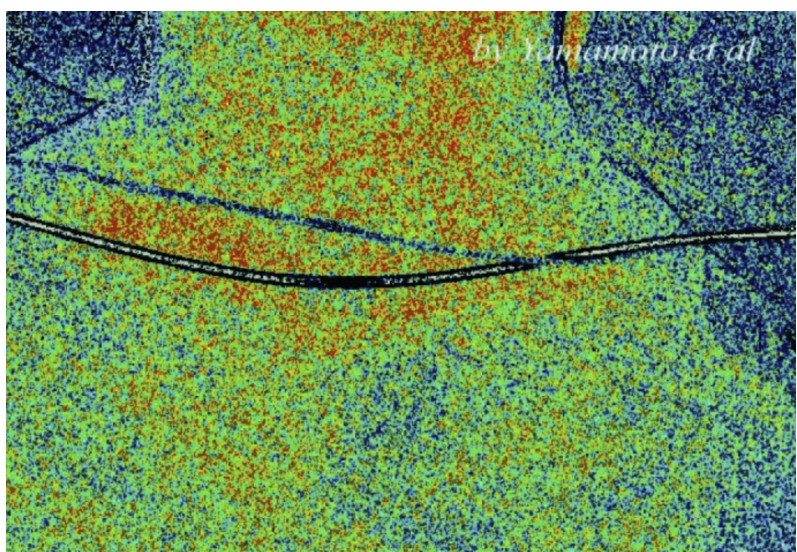

VIDEO 2. The SBP changes are identified intraoperatively by changes in color; red color indicates a sufficient volume of SBP. While we electrically stimulated the sixth thoracic sympathetic ganglion, a blue color in the left subclavian area appeared transiently, indicating a decrease in SBP in that area. Video available at: http://www.jtcvsonline.org/article/S0022-5223(17)31157-1/fulltext. 

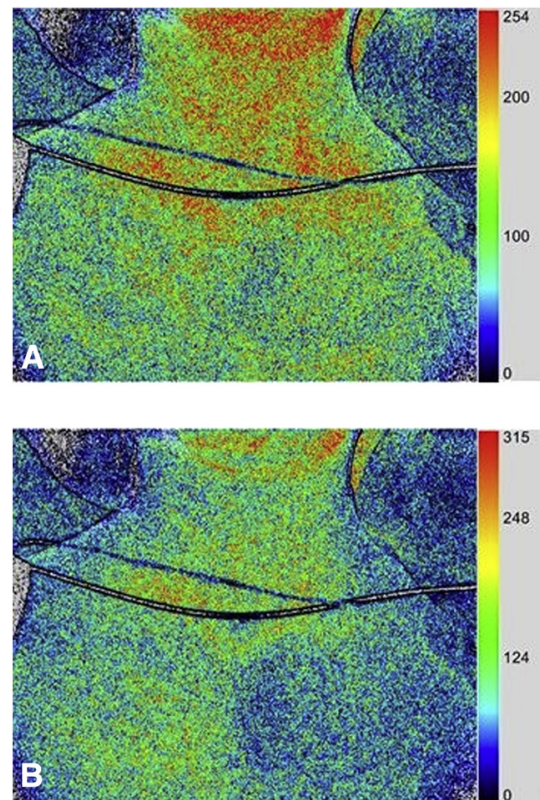

C

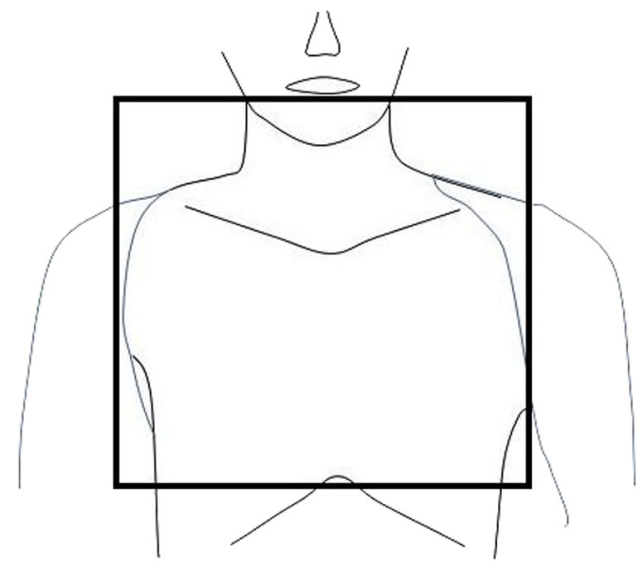

FIGURE 2. Intraoperative laser speckle flow graph. A, SBP changes are indicated by color changes; red indicates a sufficient volume of SBP. B, When we electrically stimulated the sixth thoracic sympathetic ganglion electrically, a blue color in the left subclavian area appeared, indicating a decrease in SBP in that area. C, Image area.

in blood perfusion and sweating is induced in the corresponding area of the skin. Therefore, LSFG-guided excision of the related sympathetic nerve can treat CS.

\section{References}

1. Sugiyama T, Araie M, Riva CE, Schmetterer L, Orqul S. Use of laser speckle flowgraphy in ocular blood flow research. Acta Ophthalmol. 2010;88:723-9.
2. Lee MC, Konishi N, Fujii H. Blood flow analyses of skin tissue under the sacrum using laser speckle graphy. Optical Rev. 2003;10:562-6.

3. Shelley WB, Florence R. Compensatory hyperhidrosis after sympathectomy. N Engl J Med. 1960;263:1056-8.

4. Andrews BT, Rennie JA. Predicting changes in the distribution of sweating following thoracoscopic sympathectomy. Br J Surg. 1997;84:1702-4.

5. Lesèche G, Castier Y, Thabut G, Petit MD, Combes M, Cerceau O, et al. Endoscopic transthoracic sympathectomy for upper limb hyperhidrosis: limited sympathectomy does not reduce postoperative compensatory sweating. J Vasc Surg. 2003;37:124-8. 